\title{
Ins „Bildern“ kommen
}

\section{Zur Veralltäglichung interpersonaler Bildkommunikation}

\author{
WOLFGANG REISSMANN
}

\section{EINLEITUNG}

Dieser Beitrag beschäftigt sich mit einem Phänomen, das seit etwas mehr als einer Dekade im Kontext von Mobiltelefonie und den sogenannten sozialen Medien zur Normalität geworden ist. Es geht um die Tatsache, dass (zuerst) vor allem junge Menschen (mittlerweile alle Altersgruppen) vermehrt im Modus des Bildlichen miteinander kommunizieren, sei es über das Versenden von Bildnachrichten via Messengerservices (z.B. WhatsApp) oder das Posten von Bildern in Netzwerkplattformen (z.B. Facebook). So wie Menschen miteinander sprechen und schreiben, kommen sie offensichtlich immer häufiger auch ins „Bildern“. Es stellt sich an diesem Punkt die Frage, ob und inwiefern sich (interpersonale) Kommunikation verändert, wenn Bilder selbstverständliche Mittel und Träger von Interaktion werden. Seriös beantworten lässt sich diese Frage zum gegenwärtigen Zeitpunkt nicht - es macht aber Sinn sich mit ihr zu beschäftigen. In dieser Absicht wird in einem ersten Schritt zunächst präzisiert, in welcher Hinsicht von einer Veralltäglichung der Bildkommunikation gesprochen werden kann. Darauf aufbauend werden, ohne Anspruch auf Vollständigkeit und als Anregung verstanden, einige Überlegungen zur Veralltäglichung bildkommunikativer Interaktion formuliert.

\section{INTERPERSONALE KOMMUNIKATION MIT/IN BILDERN}

Wir leben in einer Zeit, die in starkem Maße von Visualisierungen und Bildern unterschiedlichster Art geprägt ist. Allerdings: Nonverbale Kommunikation, also das Interagieren mittels Gebärde, Geste, Mimik oder Blick, ist eine anthropologi- 
sche Konstante, und auch die sekundäre Sensualisierung der Umwelt durch Bildmedien ist nicht neu. Zahlreiche kommunikations- und medienwissenschaftliche Denkfiguren basieren - etwa im Anschluss an Jean Baudrillards Simulakra-Theoreme (1982) - auf der Unterstellung, dass Medien Surrogate der Wirklichkeit produzieren. Ebenso ist eine Ästhetisierung, verstanden als ein mit der Moderne verknüpfter Prozess u.a. des ,verstärkte[n] Eindringen[s] künstlerisch gestalteter Gegenstände in den Lebensalltag der Menschen“" (Scherke 2011: 17) und der technikund medieninduzierten Erweiterung der Wahrnehmungsmöglichkeiten, kein Prozess, der gerade erst beginnt oder uns überrollt, weil wir Telefone mit integrierten Kameras in den Händen halten, auf Facebook Bilder posten oder die Bildströme von Freund*innen, Bekannten und anderen Akteur*innen verfolgen. In der ersten Ausgabe des Journal of Visual Culture bemerkt Mark Poster (2002: 67) mit Blick auf die vielfältigen Visualisierungsdiagnosen kritisch: „Does it mean that we use our eyes more than in the past? I think not." Vielmehr müsse es darum gehen, die sich historisch unterscheidenden skopischen Ordnungen in ihren Spezifika zu analysieren.

In einem ersten Schritt ist daher zu präzisieren, inwiefern - für die digitale Gegenwart - von einer Verbildlichung der Kommunikation gesprochen werden soll. Bilder und Sprachen als exklusive und einander ausschließende Zeichensysteme zu konzipieren, ist zum tieferen Verständnis dieses Wandels vermutlich wenig hilfreich. Denn sobald wir den Blick auf die Praxis richten, d.h. auf die „en-“ Formen schauen, die Aktivitäten und Tätigkeiten des Denkens, Sprechens, Schreibens, des Bildens, Bildermachens, Bebilderns usw. haben wir es mit einem Modalitätenmix zu tun:

- weil sich „Denken“ nicht allein begrifflich-prädikativ vollzieht - Stichwort „anschauliches Denken“ (Arnheim 1972) - und selbst abstrakte Begriffe (sofern sie nicht künstlichen Sprachen der Mathematik oder Informatik entstammen) auf empirische Gehalte verweisen: Natürliche Sprachen, lehrt die Wissenssoziologie, vergegenständlichen ,gemeinsame Erfahrung“ und machen diese ,allen zugänglich, die einer Sprachgemeinschaft angehören“ (Berger/ Luckmann 2004: 72). Umgekehrt mag Erfahrung im Modus der Sprache objektiviert und tradiert werden, ist selbst aber eine irreduzibel multimodale Kategorie;

- weil Sprechen und Schreiben (wenngleich in Sprache übersetzt) oftmals gerade die Beschreibung visueller Wahrnehmung nutzt (vgl. zum Bild als Binnenphänomen der Literatur z.B. Frank 2009: 370ff.); 
- weil umgekehrt Bilder sehr unterschiedlich symbolisieren: „konkret-bildhaft“, „schematisch-bildhaft“ oder „schematisch-abstrakt“ (Schwemmer 2005; vgl. auch Langer 1987);

- weil uns die Kognitionspsychologie lehrt, dass es (vermutlich) zwar verschiedene Informationsverarbeitungswege für verbale und visuelle Stimuli gibt, unser Gedächtnis selbst aber mit abstrakten Modellen/Codes/Schemata arbeitet, die weder das eine noch das andere sind, sondern in denen die Qualitäten des Anschaulichen und des Begrifflich-Abstrakten ineinandergreifen (vgl. Geise 2011: 82ff.; Lobinger 2012: 81f.);

- weil Gebärde und Geste (sofern man diese als Vorläufer einer visuellen Kommunikation betrachten will) und Sprache phylogenetisch in der Entwicklung des Menschen einen inneren Zusammenhang bilden (vgl. Tomasello 2011);

- weil ein Bild in unseren medialen Alltagswelten selten allein daher kommt und vielmehr als multimodales bzw. -kodales Gesamtzeichen in Erscheinung tritt (z.B. Bildtitel/-untertitel, Bildbeschreibung, schriftliche Codes im Bild) (vgl. z.B. Bucher 2010);

- weil sich Handeln zeitlogisch in Sequenzen vollzieht und rekursiv ist, d.h. auf früheres Handeln Bezug nimmt: auf eine Bildpräsentation folgt z.B. eine Anschlusskommunikation, Bildproduktion ist begleitet von Nachdenken usw. (vgl. Reißmann 2015: 99f.).

Trotz aller Verbunden- und Verwobenheit macht es dennoch Sinn, gerade heute und angesichts der eingangs genannten Phänomene im Kontext digitaler Medienumgebungen über die Veralltäglichung interpersonaler Bildkommunikation und die daraus resultierenden Spezifika des Kommunizierens nachzudenken. Unabhängig von utopischen und dystopischen Auslegungen, die das Sprechen über Bilder stets begleiten, ist hierbei zunächst zu konstatieren, dass medial und bildlich konstituierte (visuelle) Umwelten als Horizonte des Handelns sowie Bilder als Vermittlungen kommunikativen Handelns sich überall im Alltag eingenistet haben. Bilder sind mehr und mehr nicht nur Gegenstände der (inneren oder äußeren) Kommunikation (z.B. gemeinsam ein Fotoalbum anschauen und darüber sprechen), sondern fungieren als Mittel der Kommunikation. Als Metapher könnte eine Art bildgestützte Pantomime dienen (wobei dieser Vergleich nicht implizieren soll, dass sich visuelle Kommunikation nur in der visuellen Umschreibung sprachlicher Bedeutungsgehalte erschöpft). Hinsichtlich nonverbaler Kommunikation sind es die bildliche Vermittlung und die rahmenden medialen Infrastrukturen, die einen Unterschied machen. In Bezug auf die historische Kontinuität von Bildern im Alltag ist es die Verbildlichung der interpersonalen Kommunikation und hierbei der Übergang von einer (sprachlichen) Kommunikation über Bilder (die fortbesteht) 
zu einer (nonverbalen) Kommunikation in Bildern. Freilich ist diese auch heute nicht der Regelfall. Gleichwohl ist sie aber auch nichts Außeralltägliches mehr, nach der man etwas bemüht im Bereich der Kunst suchen müsste und die man dort z.B. in dialogischen Ausstellungskonzepten finden würde. Noch 2002 konnte Andreas Schelske zu Recht konstatieren, dass „kaum jemand auf ein Bild mit einem Bild“ antwortet, sondern ,[i]n den meisten visuell kommunikativen Situationen, in denen Bilder verwendet werden [...] Rezipienten mit Schweigen, mit einer Geste oder mit verbalen Äußerungen“" reagieren (2002: 154). Heute ist es schon weniger abwegig, wenn auch nicht alltäglich, dass auch die Reaktion auf ein Bild in Bildern erfolgt. Die Grundlage dafür liefern digitale Infrastrukturen der kommunikativen Vernetzung, die sich in einer Mischung aus technologischer Imagination, kollektiver Aneignungspraxis, kommerziellem Kalkül, staatlich-juristischer Regulierung und Zufall herausgebildet haben. Wir leben in ,dichte [n] Medienumgebungen aus unterschiedlichsten Medien" (Krotz 2010: 108, kursiv i.O.), in denen sich das traditionelle Netz von Face-to-Face-Kommunikation zusehends mit dem ,zweiten, digital vermittelten, kommunikativen Netz“ (ebd.: 109, kursiv i.O.) verschränkt hat.

Die vernetzten Infrastrukturen der Kommunikation, über die Kinder und Jugendliche heute verfügen, bringen - neben anderem - eine neue Qualität bildlicher (Quasi-)Mobilität mit sich, die nun auch die interpersonalen Netzwerke erreicht hat. Resultat ist vielleicht nicht das von Marshall McLuhan bereits in den 1960erJahren primär am Gegenstand Fernsehen insinuierte (eine), ,globale Dorf“. Dass es allerdings schwer(er) wird, (entfernten) Anderen in der „Mediapolis“ (Silverstone 2008) auszuweichen und uns Bilder und Bildmedien in einen Zustand permanenter, taktil-synästhetischer Betroffenheit versetzen, sind Gedanken, die nicht an Aktualität verloren haben. Neu an den heutigen digitalen Infrastrukturen ist, dass der persönliche mediale Erscheinungsraum nicht mehr nur von massenmedialen Text-, Ton- und Bildströmen durchdrungen ist, sondern ebenso von den Strömen der interpersonalen Medienkommunikation, die Freunde, Bekannte und Verwandte sowie die Technologien mit ihren algorithmischen Filter- und Boost-Aktivitäten in Gang setzen. Und was zur normalisierten Alltagspraxis vieler Menschen gehört, sollte auf lange Sicht auch Spuren in der Art und Weise hinterlassen, wie gedacht, wie imaginiert und wie miteinander in Beziehung getreten wird - ein Leitgedanke der Mediatisierungstheorie (vgl. bzgl. Bildpraktiken Reißmann 2015). Wenn also richtig ist (was weiterhin zu beobachten bleibt), dass wir uns zunehmend mit dem Mittel des Bildlichen adressieren und interpersonale Kommunikation darüber wenigstens in Teilen neue Formen ausbildet, kann das nicht folgenlos bleiben für die Wahrnehmung und Artikulation von Selbst, Anderen und (Um-)Welt. 


\section{ÜBERLEGUNGEN ZUR VERALLTÄGLICHUNG INTERPERSONALER BILDKOMMUNIKATION}

Gehen wir davon aus, dass die (ggf. wechselseitige) bildkommunikative Adressierung eine selbstverständliche Konversationsform ist oder sein wird, stellt sich die Frage, was das Spezifische am „Bildern“ ist und wie es sich vollzieht. Schlussendlich lässt sich diese Frage nur im Zusammenhang bzw. im Kontext konkreter Interaktionen beantworten. Es liegt auf der Hand, dass es zu Hybridisierungen der Basismedien kommt, d.h. Bild, Wort und Zahl gemeinsam zeichenwirksam werden. Und natürlich verdrängen Bilder auch nicht einfach Worte, sondern sind - als unausgesprochene Kommunikate - vielfach gerade Anlass für (sprachliche) Anschlusskommunikation. Dennoch sollten wir intensiver darüber nachdenken, was es eigentlich heißt, wenn Bilder an die Stelle von Worten und Sätzen rücken. Hierzu werden nachfolgend, ohne Anspruch auf Vollständigkeit und Trennschärfe, sieben Überlegungen dargestellt (vgl. eingehender dazu Reißmann 2015). Die ersten reflektieren die Wahrnehmungsnähe und den präsentischen Charakter, welche die Bildkommunikation kennzeichnen. Die weiteren fragen nach der Basis eines bildkommunikativen (Fremd-)Verstehens und nach der Möglichkeit, Artikulation bildlich zu individuieren.

\section{Quasi-physische Präsenz}

Wer sich Phänomenen der Medienkommunikation aus einer handlungsorientierten Perspektive nähert, rückt für gewöhnlich Fragen der (sprachnahen/-analogen) Zuschreibung, sozialen Verhandlung und (teil-)kulturellen Kontextualisierung von Bedeutung in den Vordergrund. Weil zunehmend mit Bildern kommuniziert wird, ist es erstens wichtig, neben inneren und äußeren Verstehens- und Kommunikationsprozessen der Versprachlichung und der Bezeichnung von Bedeutung auch die ästhetischen Dimensionen der Kommunikation stärker zu berücksichtigen: Bilder wollen betrachtet und nicht nur interpretiert werden. Die Bildphilosophie diskutiert diesen Antagonismus in der Kontroverse zwischen semiotischen und wahrnehmungsphänomenologischen Positionen (vgl. Sachs-Hombach 2006: 82ff.). Wahrnehmungsnähe ist unbestreitbar ein Merkmal jeder bildlichen Kommunikation, auch wenn, wie eingangs argumentiert, die Grenzen nicht hermetisch an den materialisierten Ausdrucksformen und den Grenzen von Sprache und Bild zu ziehen sind. Als (quasi-)materielle Umgebungen und Umwelten sind Bilder nicht nur Flächen und Räume, die wir propositional auf ihren Sinn befragen, sondern Wahrnehmungs- und Erfahrungsräume. Blenden wir ihre kommunikative Einbindung (also die Tatsache, dass irgendwer irgendetwas artikulieren bzw. zeigen 
möchte) für einen Moment aus, blicken wir in Bildern auf absente bzw. repräsentierte Räume oder auf Dinge, Figuren/Personen, Farben, Formen, Muster und anderes mehr. Dinge und Körper in einem Bild zu sehen, schließt dabei an die Erfahrung und visuelle Orientierung im „Real“-Raum an. Zugrunde liegen kindliche Entwicklungs- und Sozialisationsprozesse, die bspw. entlang Meads Theorie der Dingkonstitution als eine Erweiterung der Widerstandserfahrungen der Dinge (,pushiness“) vom taktilen auf den visuellen Raum beschrieben werden können (vgl. Zirfas/Jörissen 2007: 67f.).

So wie wir Fern-Dinge als „echte“ Dinge wahrnehmen und wir dabei auch ohne Realitätstest am Gegenstand ein Gefühl für ihre Schwere oder Beschaffenheit haben, so ist es uns möglich, bildlich repräsentierte Dinge und Körper als solche wahrzunehmen. Vor allem für zentralperspektivisch und naturalistisch gestaltete Bilder liegt in Anlehnung an Leon Battista Alberti (als Vorbereiter der Zentralperspektive) die Metapher des „offenen Fensters“ nahe (vgl. Snyder 1980). Selbstredend stößt diese an Grenzen, sobald Medialitätsbewusstsein unterstellt wird oder Bilder intransparent, opak oder nicht-reproduzierend gestaltet sind. In einem schwachen Sinn bleiben Parallelen zur unvermittelten visuellen Wahrnehmung aber auch dann konstitutiv. Eine Kommunikation mit Bildern legt daher (quasi-)physische Präsenzerfahrungen nahe (obgleich wir uns natürlich auch distanzieren können und uns die Imaginationskraft solche Erfahrungen auch im mündlichen Gespräch oder beim Lesen ermöglicht). Bilder zeigen nicht nur ihre Bedeutungen hervor, sondern bieten den Betrachtenden Welten an, in denen diese sich imaginär (synästhetisch) bewegen können. Diese Bewegung ist nicht primär und allein ein kognitiv-kommunikatives Entziffern, sondern ebenso ein (Nach-) Erleben und Spüren von Dingen, Formen, Konstellationen, Größen, Perspektiven, Farben usw. Diese in der visuellen Umwelt gemachten (sinnlichen) Erfahrungen können im Kontext von Verstehen und bildbezogener Interaktion wieder (innerlich/äußerlich) sprachlich oder anderweitig vergegenständlicht und auf diese Weise nachträglich kommunikabel gemacht werden.

\section{Teilhabe und Perspektivübernahme}

Nehmen wir die kommunikativen Kontexte wieder mit hinein (also die Tatsache, dass irgendwer irgendetwas artikulieren bzw. zeigen möchte), sensibilisiert die Beschäftigung mit Ästhetik und Bildlichkeit zweitens dafür, dass es bei der Perspektiv- und Rollenübernahme als wichtiger Grundlage zwischenmenschlicher Kommunikation und Interaktion (vgl. Mead 1973) nicht nur um propositionalkognitive Antizipationen des Gegenübers geht. Es wird nicht nur aus der Warte des kommunikativen Gegenübers gedacht, bewertet oder Handlungsfolgen antizipiert, sondern ebenso - multimodal und (syn-)ästhetisch - gesehen, gehört, getas- 
tet, sich bewegt, gespürt usw. Unabhängig davon, was sich an reflexiver Bedeutungsgebung anschließt, eröffnen Bilder einen Zugang zu Welten, die andere (re-) präsentieren. In dieser Hinsicht können wir Bilder auch als arretierte Blicke eines (zeigenden) Kommunikationspartners begreifen. Diese Blicke sind freilich nicht „unschuldig“, sondern das Ergebnis vielfältiger kultureller und medialer Formungen (was wiederum Anschluss schafft für die differenzsoziologischen Programme der Geschlechtlichkeit, der Herkunft, der habituellen Distinktion usw.). In der Praxis müssen sie zudem (meta-)kommunikativ qualifiziert werden: als ,so und nicht anders“ oder ,,augenzwinkernd“ gemeint oder ausgewiesen werden als eigene oder fremde „Blicke von Dritten“, die man voll und ganz, partiell oder auch nicht teilt.

Diese Qualifizierungen vollziehen sich nur zum Teil über Bildlichkeit, sondern wesentlich auch über (zumeist sprachliche) Kontextualisierung oder auch implizit über das kommunikative Umfeld. Auf einer basalen Ebene aktualisiert sich in der Bildrezeption jedoch die Erfahrung, dass das gleiche Bild bereits von (einem oder mehreren) Anderen gesehen worden sein muss und nicht nur im übertragenen Sinne gleichsam einen Standpunkt/eine Perspektive verkörpert. Auf diese Weise werden aus dem bloß bildlich vermittelten Präsenzerleben ein geteilter Erfahrungsraum und ein geteilter Beobachterstandpunkt, weil nicht nur Ego, sondern gleichsam auch Alteri imaginär präsent sind und mitschauen (auch wenn das in actu nicht ständig bewusst ist und nicht immer konkrete Andere auszumachen sind). „Geteilt" impliziert hierbei keine Konsensfiktion, sondern eher das Erleben gemeinsamer Anwesenheit, die Teilhabe an einer gemeinsamen Situation.

\section{Vergegenständlichung von Erfahrung und Erleben}

Die heute vielfach gegebene Möglichkeit, Eindrücke eines jeden Hier und Jetzt seien diese auf eine „,innere“ oder auf eine ,äußere Realität“ bezogen - ohne den Umweg sprachlicher Übersetzung u.a. mittels Bild und Fotografie zu vergegenständlichen und durch mediale Infrastrukturen (instantan) zu distribuieren, geht drittens mit der Aufwertung einer nichtsprachlichen Objektivation von Erfahrung als Mittel der Kommunikation einher. Das Artikulationsprinzip selbst ist dabei nicht neu. Werke der bildenden Kunst beruhen ebenso darauf wie es in den Alltagspraktiken von Kindern und Jugendlichen eingelassen ist, z.B. kindliche Artikulationen durch Malen und Zeichnen, szenespezifisches Bildhandeln im Kontext von Graffiti und Visual Streetart oder die Fotografie als jugendliches Ausdrucksmedium im Kontext von Reisen, Festen, Beziehungen, Foto-Wettbewerben u.a.m.

Gerade Medien- und Kunstpädagogik haben in zahlreichen Projekten gezeigt, dass präsentative Medien genutzt werden, um Vorstellungen, Emotionen, Lebensgefühle, soziale Räume, Lebenswelten oder kulturelle Verortungen zur Anschau- 
ung zu bringen (vgl. stellvertretend Holzwarth 2009). Oft wird darauf verwiesen, dass mit Bildern (und anderen präsentativen Medien) artikuliert wird, was sich sprachlich nicht fassen, begreifen und beschreiben lässt. Wenn bildliche Vergegenständlichung zu einem allgemeinen Kommunikationsprinzip wird, geht es nicht mehr (nur) darum, verbale Unausdrücklichkeit zu kompensieren. Die schlichte Möglichkeit, scheinbar ohne aufwändige Übersetzungsleistungen aus dem Hier und Jetzt heraus berichten zu können und mit dem Bild eine alternative Ausdrucksquelle zur Hand zu haben, ist Grund genug, sich dieses Mediums zu bedienen. In diesem Sinn können bildmachende, -verbreitende und -zeigende als mimetisch Agierende und (sich und andere/s) Zitierende verstanden werden. In Anlehnung an Gunter Gebauer und Christoph Wulf bezeichnet Mimesis die „wiederholende Herstellung vorgängiger Welten“, „,in der Menschen diese noch einmal als ihre Welten machen, aber nicht mit Hilfe des theoretischen Denkens, sondern mit Hilfe der Sinne, also aisthetisch“" (2003: 8, kursiv i.O.).

Überführt und eingebunden in Kommunikationszusammenhänge werden diese bildlich vergegenständlichten Welten zur Adressierung genutzt. In bildkommunikativen Zusammenhängen geht es vielfach darum, individuelle und kollektive sowie gegenwärtige und vergangene Erfahrung und Erleben (wieder) zu vergegenwärtigen, im Wortsinn darzustellen und präsent zu machen und Anderen vor Augen zu führen. Darstellen reduziert sich hierbei nicht auf pseudo-realistisches Abbilden als einem weiterhin zentralen und kulturell eingeschliffenen Modus des Umgangs mit fotografischen und anderen Bildern, die auf Bildkulturen der Indexikalität Bezug nehmen. Als arretierte Blicke können sie sich auf Unterschiedliches beziehen: auf eine ,äußere Wirklichkeit“, auf Vorstellungsbilder als Ausdruck subjektiven Innen- und Gefühlslebens oder kollektiven Erlebens; oder Bilder wenden den Blick als Mittel des dramaturgischen Handelns auf die Zeigenden zurück. Ebenso wird im Konjunktiv gebildet oder werden parallele und alternative Realitäten zur Anschauung gebracht.

\section{Interpersonal vernetzte Bildströme}

Digitale Infrastrukturen der kommunikativen Vernetzung führen viertens dazu, dass Menschen, wenn sie das wollen und zulassen, permanent in Verbindung stehen (mithin fragt Technik allerdings auch nicht nach ihrem Einverständnis). Zur Umschreibung dieses Phänomens haben sich Metaphern wie „Always-on“ oder der Begriff der kommunikativen/konnektiven Präsenz etabliert. Nick Couldry spricht von „Gruppen-Liveness“ als einer kontinuierlichen Vermittlung von Gruppen, die ,über den gemeinsamen Zugang zu einer Kommunikations-Infrastruktur, deren Zugangspunkte selber mobil sind“ (2006: 114) wenigstens dem Prinzip nach dauerhaft geöffnet und erreichbar bleiben. Die dezentrale Struktur vielfach 
verflochtener egozentrierter Netzwerke (als basale Strukturierung z.B. in Microbloggingdiensten oder Netzwerkplattformen) erhöht die Konnektivität bzw. technische Erreichbarkeit zwischen Freund*innen, Bekannten und Fremden sowie zwischen singulären und institutionalisiert-kollektiven Akteuren. Eine Folge davon ist, dass Individuen und Kollektive vielfältige Bildströme produzieren (und diesen ausgesetzt sind), die über diverse Devices, Interfaces und algorithmisch gesteuert (für Laien und User in ihrer Logik nur in Ansätzen durchschaubar) in Profilen zusammenlaufen und in variierender Form z.B. als Newsfeed oder Timeline sichtbar gemacht werden.

In sozialräumlicher Hinsicht kann sich vor diesem Hintergrund ein Erleben einstellen, das sich als multilokale Präsenz kennzeichnen lässt (vgl. Reißmann 2014a: 97f.). Es wird möglich, zumindest suggerieren Technik und Bilder dies, dass wir - parallel und je nach Veröffentlichungsmodus ggf. auch synchron - am Leben und Alltag von Freund*innen und Bekannten, aber auch von Stars, Marken oder Firmen und deren Events teilhaben können. Wahrnehmungsnahe Bilder, vor allem Fotografien, stützen hierbei das Erleben von Quasi- oder Als-ob-Präsenz. Gehen wir zudem davon aus, dass Beziehungspflege infolge ihrer Mediatisierung heute einen prinzipiell translokalen Charakter aufweist, kann das Partizipieren an (resp. das Ausgeschlossensein oder das Versiegen von) Bildströmen aus dem Freundes- und Bekanntenkreis in geradezu existentieller Weise mit Wohlbefinden und dem Gefühl „ontologischer“ (Un-)Sicherheit verquickt sein, wenn man darunter ein Vertrauen auf lebensweltliche Stabilität und soziales Eingebundensein versteht (vgl. Reißmann 2014b). Es ist jedenfalls alles andere als sinn-los, wie manche Erwachsene sich empören, dass Jugendliche sich vis-à-vis treffen und letztlich vermeintlich doch nur von ihren Bild- und Textwelten absorbiert zu sein scheinen. Nähe ist in einer Welt der (Bild-)Ströme eine (noch) komplizierte(re) Kategorie geworden.

\section{Motivische und ästhetische Standardisierung}

Die bis hierhin unterbreiteten Überlegungen sind primär vom Gedanken geleitet, dass eine interpersonale Bildkommunikation beständig Quasi-Umwelten schafft, die von Kommunizierenden dargestellt, verbreitet und rezeptiv (imaginär) exploriert, durchschritten und erlebt werden. Wahrnehmungsnähe prägt aber auch die Vermittlung von Bedeutungen, die Bilder in Kommunikations- und Interaktionsprozessen haben (sollen), und die diesen implizit oder explizit von den beteiligten Parteien zugeschrieben werden.

In der Literatur werden Bilder oft als besonders bedeutungsoffen und polysem behandelt (was grundsätzlich indes für alle Medientexte und nicht weniger für Sprache gilt). Trotz der prinzipiellen Unmöglichkeit vollständigen Fremdverste- 
hens bleibt zwar nicht in allen, aber in zahlreichen kommunikativen Zusammenhängen eine zentrale Erwartung alltäglicher kommunikativer Adressierung, dass wir als Zeigende und Betrachtende zumindest den (Haupt-)Sinn des Gezeigten in annähernder Übereinstimmung erfassen können. Daher geht, so die fünfte Überlegung, die Veralltäglichung von Bildkommunikation vielfach mit Prozessen der motivischen und ästhetischen Standardisierung einher. Auch dieses Prinzip ist aus der Symbol-, Bild-, Fotografie-, Kunst- und Medientheorie bekannt. Immer wieder erfahren konkrete Motive und Bilder, aber auch typisierte Ereignisdarstellungen und bestimmte Symbole durch wiederholte Verwendung und (teil-)öffentliche Zirkulation eine Aufladung mit primären Bedeutungsgehalten. Einige davon werden zu Ikonen, die symbolisch z.B. für historische Ereignisse, technischen Fortschritt, das Lebensgefühl einer Generation usw. stehen (vgl. Paul 2009).

Spätestens seit Aby Warburg gibt es in der Kunstgeschichte das Bemühen, Konstituenten von Bildsprachen zu identifizieren. Warburg beschäftigte sich mit „Pathosformeln“ bzw. der Darstellung von Affekt und Gebärde, v.a. vergleichend in Antike und Renaissancemalerei. Einen anderen Zugang unternahm Otto Neurath. In den 1930er-Jahren bemühte er sich um die Entwicklung einer internationalen „Bildsprache“ auf der Basis intuitiv zugänglicher Piktogramme. Das Gelingen solcher Vorhaben, analog zur Sprachanalyse „kleinste bedeutungstragende Elemente einer Bildsprache“ (Knape 2008: 121) aufzuweisen, darf jedoch bezweifelt werden. Dafür fehlt der (reinen) Bildkommunikation die kombinatorische Grammatik der natürlichen Sprachen (vgl. in Rekurs auf Goodman Sachs-Hombach 2006: 100ff.). Gleichwohl ist alltagspragmatisch von weicheren, kontextgebundenen Konventionalisierungen primärer Bedeutungsgehalte auszugehen.

Grundsätzlich gilt, dass Bilder Figuren, Dinge, Formen, Muster, Farben oder Flächen in einem vorgegebenen Wahrnehmungsfeld (Kadrierung) simultan zur Anschauung bringen. Aus diesem simultanen Organisationsprinzip begründen sich Prägnanz- und Wiedererkennungseffekte, wenn Elemente (für die Rezipient*in) in gewohnter Weise versammelt werden und auf eine Stabilisierung von Wahrnehmungsmustern aufgebaut werden kann. Aus diesem Organisationsprinzip begründen sich aber auch Prägnanzbildungen, die auf den Bruch mit Wahrnehmungsroutinen und/oder auf eine kontrastierende und vergleichende Wahrnehmung spekulieren. Eine Art Kreislauf ergibt sich, wenn diese als Irritationen und Abweichungen entworfenen Artefakte selbst wiederum in den Bereich der Wahrnehmungsroutinen übersiedeln und in stabilisierte Wahrnehmungsmuster münden, etwa durch sich allmählich verfestigende Genreerwartungen. Wege zur Stabilisierung von Bildbedeutung liegen z.B. in pointierten, dramaturgisch gesteigerten und/oder reduzierten Darstellungsweisen; in kulturellen Ikonisierungsprozessen, die Bilder erfahren können, ohne zuvor auf Prägnanz hin gestaltet worden zu sein; sowie 
damit zusammenhängend und vielleicht das wichtigste Prinzip: in der Redundanz. Der beständig wiederholte Einsatz bzw. Gebrauch bestimmter Bilder und Bildtypen in vergleichbaren Kommunikationszusammenhängen ist ein wichtiges Moment für die überindividuelle Stabilisierung von Bildbedeutung.

Populär- und Medienkultur hat (teils globalisierte) „Bildsprachen“ hervorgebracht, die sich z.B. über Film- und Plakatästhetik, über Starinszenierungen oder Visiotype (Pörksen 1997) etabliert und ausdifferenziert haben. Nicht weniger als im massenmedialen Bereich sind motivische Wiederholung, stilistische Konstanz und Wiedererkennbarkeit aber auch historische Kennzeichen der Privatfotografie und so genannten „Kodak Culture“ (vgl. Reißmann 2015: 125ff.). Insofern Bildkommunikation an Bedeutung zunimmt, kann daher nicht überraschen, dass schematisch anmutende Wiederholungen des scheinbar immer selben dominieren. Dieses Prinzip der Verdichtung von Bedeutung und des Typisierens ist allerdings nicht gleichzusetzen mit Fragen und Phänomenen der Stereotypisierung, die darauf auch aufbauen, aber weitere Implikationen enthalten.

\section{Individuierung bildlicher Artikulation}

Insofern in einem schwachen und metaphorischen Sinn dahingehend von einer Art Bildsprache gesprochen werden kann, dass bestimmte Motive sowie Arten und Weisen der Darstellung mit primären, überindividuell verständlichen Bedeutungsgehalten aufgeladen sind (wenngleich sich diese im Zeitverlauf auch wieder verschleifen; vgl. Faßler 2002: 95), stellt sich sechstens die Frage, wie dieses symbolische Vokabular in Kommunikationssituationen für individuelle, d.h. an bestimmte (ggf. kollektive) Akteur*innen gebundene „Aus-Sagen“ respektive „AusZeigen“ genutzt und gleichsam personalisiert werden kann. Zumindest für die hier im Mittelpunkt stehende zwischenmenschliche Interaktion liegt eine der zentralen Voraussetzungen wohl in der Verbindung, die alltagspragmatisch zwischen den Bildern und denjenigen, die sie zeigen, respektive denjenigen, die sie z.B. in netzwerkartig organisierten medialen Infrastrukturen passieren lassen, gezogen wird. In Prozessen der (modernen) Bildsozialisation wird nicht nur die Annahme kultiviert, dass zu Bildern Schöpfer*innen gehören, die sie hervorbringen (ink1. Apparaten), sondern auch die Annahme, dass diejenigen, die sie im Wortsinn hervorzeigen, mit Bildern etwas aus-zeigen möchten.

Die Geste des Zeigens weist, wie der Fingerzeig, imaginär genauso vom Körper weg wie auf die Zeigenden. Gehen wir davon aus, dass diese alltagspragmatischen Unterstellungen Teil des praktischen Wissens sind, das wir an Bilder herantragen bzw. das Bilder unwillkürlich in uns evozieren. Ergänzen wir noch die Annahme, dass immer auch die Möglichkeit besteht, auf etwas anderes zu zeigen und etwas anders darzustellen, das gezeigte Bild also immer nur das realisierte 
aus einer imaginären Reihe unendlich vieler Bilder ist, dann kann dies im Hinblick auf die Individuierung der Artikulationen die kommunikationstheoretische Bedeutung der Auswahl und des Stils der gezeigten Bilder begründen (vgl. allgemein zu Stil als kommunikativem Mittel Meier 2014; Reißmann 2015: 77ff.).

Der Bezug des Gezeigten zum Zeigenden qualifiziert sich darüber, was aus einem potenziell möglichen Bildspektrum gewählt wird und wie das Ausgewählte wiederum stilistisch beschaffen ist. Stil und Habitus geben zwar in jeder Form der Kommunikation (implizite) Hinweise auf die Kommunikatoren und das Gemeinte. In der mündlichen Rede individualisieren etwa Intonation, Stimmmodulation oder Wortwahl die denotativen Gehalte. Auch das kommunikative Umfeld legt Assoziationen und Zuschreibungen nahe. In einer Kommunikation mit Bildern sind Auswahl und Stil jedoch schon deshalb konstitutiv, da die zahlreichen, Ich-Bezug ausdrückenden Relationen der Sprache (z.B. das Personalpronomen „ich“, das Reflexivpronomen ,mich“) fehlen, um im Akt des Zeigens zugleich auch der eigenen Haltung zum Gezeigten Ausdruck zu verleihen. Durch die Motivauswahl und die Art und Weise der Gestaltung und Formung des sinnlichen Materials werden (bewusst und unbewusst) Konnotationsrichtungen festgelegt und dem Gegenüber spezielle Assoziationsräume nahegelegt. Setzt man dieses Kommunikationsprinzip zusätzlich in Beziehung zu individualisierungstheoretischen Überlegungen, dann überrascht es nicht, dass neben Wiederholung und Wiedererkennbarkeit (s.o.) die Produktion stilistischer Differenz und Abweichung ebenso ihren Platz im bildlichen Alltagshandeln nicht nur von Kindern und Jugendlichen hat.

\section{Variation und Bildtransformation}

Bilder und allzumal digitale Bilder laden dazu ein, gestaltet zu werden. Die kulturell stabilisierte Trennung zwischen technisch scheinbar reproduzierenden (,indexikalischen") Bildern der Fotografie und des Films (sei es im Bereich des Realistischen oder des Fantastischen) und entwerfenden Bildern (Zeichnungen, Skizzen, Pläne usw.) ist durch die Möglichkeiten und die Veralltäglichung der digitalen Bildbearbeitung durchlässiger geworden.

Um Abbildtheorien und Wahrnehmungsmodelle soll es hier jedoch nicht gehen. Vielmehr soll siebtens die für die alltäglichen Anwender*innen gestiegene Plastizität des digitalen Bildes als ein Schub für die Veralltäglichung ,echter“ Bilddialoge reflektiert werden, die im Sinne einer interpersonalen Bildkommunikation auf Sprache und andere Ausdrucksmittel verzichten können. Unter Bilddialog wird hier verstanden, dass die Reaktion auf das Zeigen eines Bildes keine „AntWort“, sondern ein „Ant-Bild“ ist. Für solche Bilddialoge braucht es keine spezielle Plastizität als Voraussetzung. Jemand kann imaginär ein Bild A hervorzeigen, das ein/e andere/r veranlasst, ein Bild B zu zeigen usw. Die in Reichweite beste- 
henden Bildarchive sind das kommunikative Arsenal, aus dem solche Bildspiele schöpfen. Sie können dadaistische Züge annehmen, wenn scheinbar zusammenhangslos Material auf Material folgt - und dabei dennoch elementar sinnvoll sein: weil der Verlauf vielleicht überrascht oder eine vergleichende Wahrnehmung einsetzt, die trotz Kontingenz und Heterogenität Gemeinsamkeiten entdeckt oder sich ihren eigenen Reim aus den Kontrasten macht.

Bilddialoge können ebenso aber auch zu Sammlungen oder rekursiven Ketten stilistisch oder motivisch ähnlicher Bilder führen. Grundsätzlich sind allerdings - maximal wie minimal - Variation und Bildvielfalt Voraussetzungen für Bilddialoge, wenn wir unterstellen, dass Akteure in Konversationen etwas zu berichten haben wollen. Freilich kann das „Ant-Bild“ ebenso wie die „Ant-Wort“ das Gesagte bzw. Gezeigte hypothetisch bloß wiederholen. Es ist aber eher unrealistisch, dass unsere Reaktion auf ein Bild, das uns z.B. ein Freund schickt, exakt das gleiche Bild ist (zumindest, wenn wir in der Dialogfigur bleibend davon ausgehen, uns mit diesem zu unterhalten, und nicht etwa seine Bilder in andere Konversationen einzubringen). Die digitale Plastizität, aber auch generell die Gestaltbarkeit von Bildern (z.B. durch Perspektive, Kadrierung und soziale Choreografie in der Fotografie) sind wichtig für Bilddialoge, da Bildpraxis immer Bezug auf Vorund Ausgangsbilder nimmt. Jedes Bild, das gemacht wird, ist in gewisser Hinsicht die (schlechte) Kopie eines oder mehrerer anderer Bilder, die uns, bewusst oder nicht, imaginär oder materiell vergegenständlicht, bereits vertraut sind. Umgekehrt ist jedes neue Bild qua Konkretion und Kontextualität einzigartig. Plastizität und Gestaltbarkeit befördern, so die These, solche Bilddialoge bzw. -ketten, die gleichermaßen von Wiedererkennbarkeit und Gemeinsamkeiten wie von Befremdung und Überraschungsmomenten leben.

Anschauungsunterricht für das kompositorische Spiel mit Vor- und Ausgangsbildern bieten gegenwärtig Meme im Internet. Alltagssprachlich werden unter dem wissenschaftlichen Begriff „Mem“ seit der letzten Dekade auch bildliche, bildtextliche und audiovisuelle populärkulturelle Artefakte gefasst, die sich via digitaler Infrastrukturen „,viral“ verbreiten und binnen kurzer Zeit sehr viele Menschen erreichen und/oder von diesen weiter geleitet und/oder transformiert werden. Ein wesentliches Gestaltungsprinzip ist das Spiel mit Inkongruenzen, die auf unterschiedliche Weise entstehen können: materiell, z.B. über das kontrastive Arrangement von Bildelementen und das Erzeugen von Bild-Text-Scheren; symbolisch/ ideell z.B. durch die Bezugnahme auf Vor-Bilder (die im einzelnen Mem selbst nicht zu sehen sind) und damit auf das Vorwissen und den imaginären Abgleich verschiedener Bilder durch die Betrachtenden. Typisch sind bspw. Text-BildReihen, in denen entweder der Text oder das Bild konstant gehalten werden und das jeweils andere Element variiert (vgl. Davison 2012: 127ff.). Solche Reihen 
bzw. Remix-Spiralen können aber auch primär oder allein visuell-bildlich erzeugt werden, wie etwa die populären Merkel-Rauten oder die Adaptionen der SituationRoom-Ikone demonstrieren. ${ }^{1}$ Nicht alle, aber viele Meme beziehen ihren Schauwert aus kompositorischen Variationen, die mit Wahrnehmung und Assoziationsfeldern spielen.

Meme sind nur ein Beispiel für umfassendere Phänomene. Die Veralltäglichung von Bildkommunikation führt dazu, dass Bilder konkret oder ideell nicht nur auf andere (Vor-)Bilder Bezug nehmen (was Voraussetzung für Bildpraxis und Grundlage für oben genannte ästhetische und motivische Standardisierungen ist), sondern in dieser Bezugnahme auch permanent transformiert werden. $\mathrm{Zu}$ diesen Transformationen zählen selbstverständlich nicht nur Bildbearbeitungen, sondern ebenso auch Abweichungen in typischen sozialen Choreografien, das Ausprobieren alternativer Perspektiven, Szenarien usw. Bildakteure produzieren damit immer auch kommunikative Perspektiven auf ein gemeinsames Thema, eine Situation oder ein Ereignis. Gerade wenn es im Kontext interpersonaler Kommunikation darum geht, mit und in Bildern zu kommunizieren, braucht es Möglichkeiten, sich differenziert(er) und bezogen auf einen gemeinsamen Gegenstand auszudrücken. In diesem Sinn stützen - vergleichsweise einfach zu bewerkstelligende - Bildtransformationen die Möglichkeit, tatsächlich mit dem Mittel des Bildes zu interagieren.

\section{REKONTEXTUALISIERUNG}

Die vorgebrachten Überlegungen zur Veralltäglichung interpersonaler Bildkommunikation setzen eher abstrakt und kommunikationstheoretisch an. Gesellschaftsund kulturtheoretische Tiefe gewinnen entsprechende Analysen aber erst (zurück), wenn die tatsächlichen, von Mensch und Technik hervorgebrachten und miteinander aufgeführten Bildspiele in den Blick geraten. Es stellt sich im Anschluss also die Frage, in welchen Kontexten und Situationen von welchen Akteur*innen welche Bilder - als Erlebnisräume/Quasi-Umwelten, arretierte Blicke/verdinglichte Perspektiven, individuierte Artikulationen, Teil von Strömen usw. - gezeigt und gestaltet werden, wie darauf wiederum reagiert wird und welche Bildketten und -reihen sich schlussendlich ergeben. Auf dieser Ebene sind auch all die „DoingFragen" einzubringen, die auf die (Re-)Präsentation und Performanz von (Teil-) Kulturen, Identitäten, Geschlechterbildern, Milieus oder Szenen im Spannungsfeld von Typisierung und Stereotypisierung, von Macht- und Herrschaftsverhält-

1 Siehe http://merkelraute.tumblr.com/; https://www.tumblr.com/tagged/situation-room [15.03.2016]. 
nissen bezogen sind oder die sich aus der Konstruktionslogik der jeweiligen medialen Infrastrukturen ergeben (Codes, Algorithmen usw.). Erst die Hinwendung zur Praxis erweist, welche heterogenen Körper- und Stil-Welten etwa Jugendliche in „Selfies“ und Portraits zur Betrachtung und Erkundung darbieten, in und aus welchen Perspektiven sie angeschaut werden sollen, in welcher Weise „Foodporn“ sinn-voll ist, wie sich Bilddialoge im Kontext von „Fitnesstracking“ und Imagestreams in Instagram ausnehmen, wie dezentral und translokal an kollektiven Bildarchiven als Gruppengedächtnissen gearbeitet wird usw.

Und schließlich bleibt bei allen Überlegungen die Diskrepanz zwischen Nutzungs- und Aneignungspotenzialen, die technologische Infrastrukturen bieten, und den differentiellen Nutzungs- und Aneignungsrealitäten zu berücksichtigen. Wenn jemand zwei Wochen lang nicht in ihren oder seinen Facebook-Account schaut, sind die Bilder, die sie/er dort zu sehen bekommt, (subjektphänomenologisch formuliert) ähnlich lang oder gar länger unterwegs - und faktisch: ähnlich (im-)mobil - wie die Bilder, die eine Person einige Jahrzehnte früher womöglich mit der Post von Bekannten zugeschickt oder bei einem Treffen bekommen hätte. Letztlich entscheidet die mediale und kommunikative Praxis darüber, wie groß die Kluft von aktuellen und vergangenen Ausprägungen von Kommunikationsmustern tatsächlich ist. Das sollte uns aber nicht davon abhalten, mögliche Tendenzen als solche zu diagnostizieren und daraufhin zu befragen, was sie für einen Unterschied machen.

\section{LITERATUR}

Arnheim, Rudolf (1972): Anschauliches Denken. Zur Einheit von Bild und Begriff. Köln:

DuMont Schauberg.

Baudrillard, Jean (1982): Der symbolische Tausch und der Tod. München: Matthes \& Seitz.

[Orig. 1976].

Berger, Peter L./Luckmann, Thomas (2004): Die gesellschaftliche Konstruktion der Wirklichkeit. 20. Auflage. Frankfurt am Main: Fischer. [Orig. 1966].

Bucher, Hans-Jürgen (2010): Multimodalität. Eine Universalie des Medienwandels: Problemstellungen und Theorien der Multimodalitätsforschung. In: Bucher, Hans-Jürgen/ Gloning, Thomas/Lehnen, Kathrin (Hg.): Neue Medien - Neue Formate. Ausdifferenzierung und Konvergenz in der Medienkommunikation. Frankfurt am Main/New York: Campus, S. 41-79.

Couldry, Nick (2006): Akteur-Netzwerk-Theorie und Medien: Über Bedingungen und Grenzen von Konnektivitäten und Verbindungen. In: Hepp, Andreas/Krotz, Friedrich/ Moores, Shaun/Winter, Carsten (Hg.): Konnektivität, Netzwerk und Fluss. Konzepte 
gegenwärtiger Medien-, Kommunikations- und Kulturtheorie. Wiesbaden: VS Verlag für Sozialwissenschaften, S. 101-117.

Davison, Patrick (2012): Language of Internet Memes. In: Mandiberg, Michael (Hg.): The Social Media Reader. New York: NYU Press, S. 120-136.

Faßler, Manfred (2002): Bildlichkeit. Navigationen durch das Repertoire des Sichtbaren. Wien u.a.: Böhlau.

Frank, Gustav (2009): Literaturtheorie und Visuelle Kultur. In: Sachs-Hombach, Klaus (Hg.): Bildtheorien. Anthropologische und kulturelle Grundlagen des Visualistic Turn. Frankfurt am Main: Suhrkamp, S. 354-392.

Gebauer, Gunter/Wulf, Christoph (2003): Mimetische Weltzugänge. Soziales Handeln Rituale und Spiele - Ästhetische Produktionen. Stuttgart: Kohlhammer.

Geise, Stephanie (2011): Vision that matters. Die Funktions- und Wirkungslogik Visueller Politischer Kommunikation am Beispiel des Wahlplakats. Wiesbaden: VS Verlag für Sozialwissenschaften.

Holzwarth, Peter (2009): Selbstausdruck mittels Medien. In: Vollbrecht, Ralf/Wegener, Claudia (Hg.): Handbuch Mediensozialisation. Wiesbaden: VS Verlag für Sozialwissenschaften, S. 446-452.

Knape, Joachim (2008): Gibt es Pathosformeln? Überlegungen zu einem Konzept von Aby M. Warburg. In: Dickhut, Wolfgang/Manns, Stefan/Winkler, Norbert (Hg.): Muster im Wandel. Zur Dynamik topischer Wissensordnungen in Spätmittelalter und Früher Neuzeit. Göttingen: V\&R unipress, S. 115-137.

Krotz, Friedrich (2010): Leben in mediatisierten Gesellschaften. Kommunikation als anthropologische Konstante und ihre Ausdifferenzierung heute. In: Pietraß, Manuela/ Funiok, Rüdiger (Hg.): Mensch und Medien. Philosophische und sozialwissenschaftliche Perspektiven. Wiesbaden: VS Verlag für Sozialwissenschaften, S. 91-113.

Langer, Susanne K. (1987): Philosophie auf neuem Wege. Das Symbol im Denken, im Ritus und in der Kunst. Frankfurt am Main: Fischer. [Orig. 1942].

Lobinger, Katharina (2012): Visuelle Kommunikationsforschung. Medienbilder als Herausforderung für die Kommunikations- und Medienwissenschaft. Wiesbaden: VS Verlag für Sozialwissenschaften.

Mead, George H. (1973): Geist, Identität und Gesellschaft. Frankfurt: Suhrkamp. [Orig. 1934].

Meier, Stefan (2014): Visuelle Stile. Zur Sozialsemiotik visueller Medienkultur und konvergenter Design-Praxis. Bielefeld: transcript.

Mitchell, William J. T. (1990): Was ist ein Bild? In: Bohn, Volker (Hg.): Bildlichkeit. Internationale Beiträge zur Poetik. Frankfurt am Main: Suhrkamp, S. 17-68.

Moores, Shaun (2006): Ortskonzepte in einer Welt der Ströme. In: Hepp, Andreas/Krotz, Friedrich/Moores, Shaun/Winter, Carsten (Hg.): Konnektivität, Netzwerk und Fluss. Wiesbaden: VS Verlag für Sozialwissenschaften, S. 189-205. 
Paul, Gerhard (2009): Das Jahrhundert der Bilder. Die visuelle Geschichte und der Bilderkanon des kulturellen Gedächtnisses. In: Paul, Gerhard (Hg.): Das Jahrhundert der Bilder. Band I: 1900 bis 1949. Bonn: bpb, S. 14-39.

Pörksen, Uwe (1997): Weltmarkt der Bilder. Eine Philosophie der Visiotype. Stuttgart: Klett-Cotta.

Poster, Mark (2002): Visual Studies as Media Studies. In: Journal of Visual Culture, Jg. 1, Heft 1, S. 67-70.

Reißmann, Wolfgang (2014a): Bildhandeln und Bildkommunikation in Social Network Sites. Reflexionen zum Wandel jugendkultureller Vergemeinschaftung. In: Hugger, Kai-Uwe (Hg.): Digitale Jugendkulturen. 2., erw. und akt. Auflage. Wiesbaden: VS Verlag für Sozialwissenschaften, S. 89-103.

Reißmann, Wolfgang (2014b): Vom „home mode“ zum ,image stream“? Domestizierungstheoretische Überlegungen zum Wandel privater Bildpraxis. In: Studies in Communication Sciences, Jg. 14, Heft 2, S. 121-128.

Reißmann, Wolfgang (2015): Mediatisierung visuell. Kommunikationstheoretische Überlegungen und eine Studie zum Wandel privater Bildpraxis. Baden-Baden: Nomos.

Sachs-Hombach, Klaus (2006): Das Bild als kommunikatives Medium. Elemente einer allgemeinen Bildwissenschaft. Köln: Halem.

Schelske, Andreas (2002): Visuell kommunikatives Handeln mittels Bildern. In: SachsHombach, Klaus/Rehkämper, Klaus (Hg.): Bildhandeln. Interdisziplinäre Forschungen zur Pragmatik bildhafter Darstellungsformen. Magdeburg: Scriptum, S. 149-158.

Scherke, Katharina (2011): Ästhetisierung des Sozialen heute und in der „Wiener Moderne“ um 1900. Zur Auflösung und neuen Verfestigung sozialer Unterschiede. In: Hieber, Lutz/Moebius, Stephan (Hg.): Ästhetisierung des Sozialen. Reklame, Kunst und Politik im Zeitalter visueller Medien. Bielefeld: transcript, S. 15-32.

Schwemmer, Oswald (2005): Kulturphilosophie. Eine medientheoretische Grundlegung. München: Fink.

Silverstone, Roger (2008): Mediapolis. Die Moral der Massenmedien. Frankfurt am Main: Suhrkamp.

Snyder, Joel (1980): Picturing Vision. In: Critical Inquiry, Jg. 6, Heft 3, S. 499-526.

Tomasello, Michael (2011): Die Ursprünge der menschlichen Kommunikation. Frankfurt am Main: Suhrkamp.

Zirfas, Jörg/Jörissen, Benjamin (2007): Phänomenologien der Identität. Human-, sozial- und kulturwissenschaftliche Analysen. Wiesbaden: VS Verlag für Sozialwissenschaften. 
